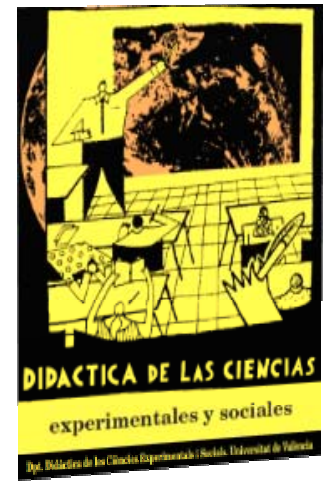

\title{
Un estudio sobre pensamiento pseudocientífico en estudiantes de educación secundaria
}

\author{
A study on pseudo-scientific thinking in high \\ school students
}

DOI:10.7203/DCES.37.15339

\author{
Guillermo Quevedo-Ortiz \\ Universidad de Granada, guiqueor@correo.ugr.es \\ Francisco González-García \\ Universidad de Granada, pagoga@ugr.es \\ ORCID iD: https://orcid.org/0000-0001-8127-9792 \\ Gracia Fernández-Ferrer \\ Universidad de Granada, gferfer@ugr.es \\ ORCID iD: https://orcid.org/0000-0002-3530-8553
}

\begin{abstract}
RESUMEN: Se presenta un estudio de la valoración y creencias de un grupo de estudiantes de Educación Secundaria sobre varias pseudociencias. Asimismo, se analizan las opiniones de los estudiantes hacia la superstición y la ciencia, y se incluye una autovaloración del grado de escepticismo. Los datos obtenidos son analizados en función del género de los estudiantes. Los resultados arrojan diferencias significativas por género al valorar algunas pseudociencias. Se realiza una propuesta de intervención educativa para $1^{\circ}$ curso de Educación Secundaria en la materia de Biología y Geología con el fin de favorecer el pensamiento crítico, basado en evidencias científicas.
\end{abstract}

Palabras clave: enseñanza de la ciencia, diferencias de género, escepticismo, pensamiento crítico, pensamiento pseudocientífico.

ABSTRACT: A study about the opinion and beliefs of a group of Secondary Education students concerning several pseudo-sciences is presented. Likewise, the beliefs of students towards superstition and science are analyzed, and a self-assessment of the degree of skepticism is included. Data obtained are analyzed according to the gender of the students. Results show significant differences by gender when evaluating some pseudosciences. An educational proposal is made for the first year of Secondary Education in the subject of Biology and Geology to favor critical thinking, based on scientific evidence.

KEYWORDS: science education, gender differences, skepticism, critical thinking, pseudoscientific thinking.

Fecha de recepción: junio de 2019

Fecha de aceptación: noviembre de 2019 


\section{INTRODUCCIÓN}

En las sociedades actuales la población está expuesta a numerosas informaciones falsas o de muy dudosa verosimilitud, mostrándose muchas de ellas en modo o con aspecto de información científica aunque en realidad no son más que ideas propias del campo de la pseudociencia. La pseudociencia puede definirse como un conjunto de ideas y/o teorías que se autodenominan como científicas pero que no cumplen los estándares científicos al no poder ser contrastadas por los métodos de trabajo habituales que utiliza la ciencia (Preece y Baxter, 2000). La pseudociencia incluye ideas muy diversas, algunas de las cuales, como la homeopatía se publicita y da a conocer por medio de grupos muy bien organizados, incluso con apoyo de diversos medios de comunicación con claros intereses comerciales (Shermer, 2003). Algunas de las pseudociencias compartieron un origen común con ciencias actuales, es el caso conocido de la química y la alquimia o la astronomía y la astrología que, aunque parten de un material de estudio común, se diferencian en el método utilizado para construir su conocimiento. Es propio de las pseudociencias no admitir el criterio de falsabilidad, esencial para la ciencia según Popper, intentando siempre esquivar las críticas o las pruebas contra sus principios (Miller, 1987).

En el pensamiento popular hay diversas pseudociencias que gozan de gran aceptación, y en ocasiones estas pseudociencias se han caracterizado como un problema pequeño y casi inofensivo, entroncando con la mitología y la literatura fantástica. Podemos citar, por ejemplo, el caso de la criptozoología, que dice estudiar la existencia de aquellos animales que la ciencia pasa por alto, ya sean tanto animales extintos como seres de fábulas y leyendas (Morrone y Fortino, 1996). Sus seguidores afirman la existencia de especies como el mamut, el mapinguarí o el yeti; y llegan a clasificar a los especímenes siguiendo la nomenclatura científica (Suberbiola y Bardet, 1998). Su falta de credibilidad se ha puesto de manifiesto al demostrarse sus trucos y manipulaciones como en caso de Nessie o en los restos del supuesto Homo pongoides (Heuvelmans, 1969) que en realidad estaba hecho de látex. Otro caso popular es la astrología en su supuesta indagación sobre el efecto y las influencias que realizan los astros sobre objetos animados e inanimados (Sabadell, 1993). Son bastante populares las predicciones sobre nuestro destino del horóscopo y carta natal, aunque ningún astrónomo podrá admitir como válidos tales vínculos (Rodríguez, 2005). Otras pseudociencias destacables son la ufología o el estudio de los OVNIS que genera gran entusiasmo entre sus seguidores (Alemañ, 2013; Gámez, 2002); la radiestesia o rabdomancia, que se encarga de detectar estímulos externos para encontrar agua, minerales, o incluso enfermedades (Azpiazu, 2004); la fisiognomía, encargada de predecir las características de las personas atendiendo a su aspecto (Caro Baroja, 1988) o la quiromancia que pretende conocer el futuro de las personas a partir de la morfología de sus manos (Webster, 2000).

Más allá de este carácter popular de las pseudociencias, algunas con claros contactos con las supersticiones, hay diversas actividades pseudocientíficas que están cobrando particular importancia por su relación con la salud humana, dejando de ser algo anecdótico para convertirse en verdaderos problemas de salud pública. En este campo es donde se está produciendo una creciente preocupación entre las autoridades sanitarias de muchos países debido al éxito de mensajes pseudocientíficos, tales como el movimiento antivacunas o ciertos tratamientos homeopáticos (Lundstrom y Jakobsson, 2009; Uskola, 2016, 2017).

Los estudios sobre las creencias y relaciones entre la ciencia y la pseudociencia son a menudo contradictorios, pues mientras hay una opinión general de que la ciencia tiene un efecto positivo en nuestras vidas y se muestra interés por la ciencia y la tecnología, a la vez se muestra interés por la pseudociencia y un cierto grado de escepticismo ante el desarrollo tecnológico. En diversas encuestas, tanto en Europa como en Estados Unidos, hay declaraciones contradictorias acerca de la ciencia, tales como "dependemos demasiado de la Ciencia y no lo suficiente en la fe" (CBS, 2002; CE, 2005; NSF, 2006). 
En España, encuestas realizadas a universitarios del Grado de Educación Primaria en Euskadi, reflejaron un desconocimiento importante sobre la homeopatía, defendiendo sus propiedades curativas (84\%) al igual que mostraron una notoria confusión con los remedios naturales (Uskola, 2016). En los Estados Unidos, se concluyó que el 36\% de la población había realizado alguna práctica alternativa en el último año, aumentando al 62\% si tenían en cuenta como terapia los ruegos y peticiones para la curación (Guarín, 2017).

La investigación educativa trabaja habitualmente en contextos complejos y con múltiples variables a considerar, así desde el campo de la Didáctica de las Ciencias Experimentales resulta de interés conocer la posible relación entre las creencias pseudocientíficas y la formación científica de la población, como un factor más a tener en cuenta en el estudio del fenómeno de las pseudociencias. Muchas encuestas de opinión pública no han encontrado relación alguna entre la formación y otras creencias de superstición popular (Goode, 2002). Preece y Baxter (2000) declaran que la aceptación creciente de las pseudociencias debe ser motivo de preocupación para los educadores científicos, de modo que un objetivo de la alfabetización científica ha de ser la diferenciación entre ciencia y pseudociencia, así como desarrollar una visión escéptica y crítica sobre las informaciones que recibimos. Diversas investigaciones señalan que estos objetivos no se alcanzan (Ryan, Brown, Johnson, Sanberg y Schildmier, 2004; Walker, Hoekstra y Vogl, 2002).

Clásicamente se indica que la alfabetización científica consta de tres aspectos: los conceptos (el conocimiento científico), los procesos científicos y las situaciones o contextos donde se desarrolla. Habitualmente la educación científica se ha centrado en demasía en el primer aspecto (solo se valoraban los conceptos), dejando un tanto al margen los otros dos aspectos. Los procesos y los contextos científicos sirven para que los individuos sean conscientes de la naturaleza de la ciencia (NOS, por sus siglas en inglés). Conocer NOS o más básicamente conocer la epistemología de la ciencia, es decir la ciencia como forma de conocimiento, sus valores y las creencias inherentes al desarrollo del conocimiento científico puede contribuir a desarrollar el pensamiento crítico y el escepticismo frente a formas no científicas de información (Abd-El-Khalick y Lederman, 2000).

En España se han realizado estudios utilizando las escalas ROSE (The Relevance of Science Education), para determinar las actitudes de los estudiantes hacia las clases de ciencias (Vázquez y Manassero, 2009; Marbà y Márquez, 2010). Preece y Baxter (2000) analizaron la influencia de las pseudociencias en centros de secundaria británicos. Sus estudios revelaron que el nivel de escepticismo aumentaba a medida que se analizaban cursos superiores, además de que encontraron diferencias en cuanto al género. Observaron un mayor nivel de escepticismo en los chicos con respecto a las chicas en la mayoría de las ideas pseudocientíficas. Según Johnson y Pigliucci, (2004) la diferencia entre chicos y chicas parece estar relacionada con qué tipo de fenómenos pseudocientíficos se estudien. En otros trabajos se ha comprobado que la edad y la formación pueden influir, en grado diverso, sobre el nivel de escepticismo (McLeish, 1984; Shermer, 2003; Wiseman y Watt, 2004).

En nuestro trabajo hemos valorado las opiniones de estudiantes de Educación Secundaria Obligatoria (ESO) acerca de seis pseudociencias, en concreto la criptozoología, la astrología, la ufología, la radiestesia, la fisiognomía y la quiromancia. Las opiniones sobre astrología y ufología son las más tratadas en trabajos de pseudociencia, no así las otras que se abordan en este trabajo (Tobacyk, 2004). Analizamos también sus propias valoraciones hacia la superstición, y las opiniones sobre el grado de credibilidad que presentan hacia la ciencia y la pseudociencia y el autoconcepto sobre su nivel de escepticismo. Además, proponemos un conjunto de preguntas a desarrollar en la asignatura Biología y Geología de $1^{\circ}$ curso de Educación Secundaria Obligatoria para fomentar el pensamiento crítico, objetivo esencial para desarrollar la competencia científica y contrarrestar el pensamiento pseudocientífico. En función de las diferencias de edad y género, descritas en la bibliografía, se ha realizado un análisis para estas variables en la población de estudiantes en que se realizó el estudio. 


\section{Metodología}

La muestra del estudio estuvo compuesta por un total de 207 estudiantes (106 alumnos y 101 alumnas) de entre 12 y 14 años, pertenecientes a dos centros de la ciudad de Granada, España, que cursaban $1^{\circ}$ ESO (113 estudiantes) y $3^{\circ}$ ESO (94 estudiantes).

Para analizar la influencia de las diferentes ideas pseudocientíficas sobre el alumnado, se utilizaron como referencia dos cuestionarios previos que versan sobre temáticas pseudocientíficas, "Revised Paranormal Belief Scale" (Tobacyk, 2004) y "Magical Ideation Scale” (Eckblad y Chapman, 1983). Estos cuestionarios, con 26 y 30 ítems respectivamente, fueron utilizados como base para diseñar y elaborar un nuevo cuestionario. Tomando como base la estructuración de estos cuestionarios, se modificaron de acuerdo con la finalidad de nuestro trabajo. De esta forma, el nuevo cuestionario constó de un total de 45 ítems en forma afirmativa, que describían diferentes temáticas del ámbito de las pseudociencias. El cuestionario modificado fue validado por 3 expertos del Departamento de Didáctica de las Ciencias Experimentales de la Universidad de Granada. En su forma final (se presenta en el Anexo 1), el cuestionario dedica 5 ítems a cada una de las seis pseudociencias (criptozoología, astrología, ufología, radiestesia, fisiognomía y quiromancia). Los 15 ítems restantes se centran en la valoración sobre superstición, 7 ítems, y sobre creencias en general hacia la ciencia y pseudociencia, otros 7 ítems; y por último, uno sobre la propia valoración del grado de escepticismo.

El conjunto de ítems fue valorado por los participantes de manera cerrada, mediante una escala Likert, la cual oscila entre "muy en desacuerdo" (1), “desacuerdo" (2), "indiferente” (3), “de acuerdo" (4) y "muy de acuerdo" (5). Una encuesta idónea en nuestro caso, consistirá en aquella en la que los ítems contienen una calificación de 1 (muy en desacuerdo) ya que hacen referencia a afirmaciones pseudocientíficas, mostrando en tal caso un importante grado de pensamiento crítico, a excepción de la última que es una valoración personal. Para la cumplimentación de los cuestionarios se pidió la colaboración de profesorado en activo de los centros participantes. De tal forma que se controló y supervisó la participación del alumnado en el cuestionario.

El estudio de los resultados se llevó a cabo tomando cada ítem como una variable continua, obteniéndose la media ponderada de 1 a 5 . Para analizar si existía relación significativa entre las diferentes respuestas hacia los ítems y las variables curso y género, se utilizó el programa estadístico IBM SPSS Statistics 23. Las diferentes medias fueron analizadas mediante la prueba estadística t de Student para muestras independientes, con normalidad. El nivel de significación utilizado fue de un p valor de 0,05. Para confirmar que no existían datos u errores anómalos se contrastó mediante la prueba de Levene para igualdad de varianzas. También se analizaron las medias de grupos de variables de la misma temática en función del género con el fin estudiar el patrón general descrito por chicos y chicas.

Tras realizar el cuestionario, se propuso y realizó un planteamiento didáctico enfocado a los alumnos de $1^{\circ}$ ESO, de 12 años, en el cuál debían responder a seis cuestiones. Las cuestiones se realizaron en la asignatura de Biología y Geología con el fin de fomentar la búsqueda de información con criterios científicos y poner en cuestión y de forma crítica diversas afirmaciones o hechos planteados por las pseudociencias. Los alumnos trabajaron en grupos (siete) de tres estudiantes. Se pusieron en práctica durante seis semanas en uno de los centros donde se aplicó el cuestionario. La actividad 1 pedía buscar información científica contrastada sobre descubrimiento de nuevas especies; la actividad 2 cuestionaba sobre la credibilidad que merecen los horóscopos; la actividad 3 y 4 interrogaba sobre comportamientos relacionados con la salud; la actividad 5 preguntaba acerca de los medios de comunicación como fuente de información y la actividad 6 trataba sobre una idea tradicional en la superstición, cuestionándola desde el pensamiento crítico que proporciona el conocimiento matemático.

En conjunto se busca cuestionar las soluciones que presentan las pseudociencias, desarrollando el pensamiento crítico. En las actividades se incluyen habilidades de pensamiento 
relacionadas con la reflexión y argumentación, búsquedas en internet u otros medios de manera autónoma, problemas numéricos y de razonamiento, y exposición de opiniones. Todos fueron realizados mediante el aprendizaje colaborativo y la resolución de problemas.

\section{RESULTADOS}

En la exposición de resultados se presentan diferentes gráficos (Gráficos 1 a 5) en los que se muestra cada una de las variables dependientes en función del género, observándose de diferente coloración, azul para los niños (hombre) y rojo para las niñas (mujer).

\subsection{Cuestionario sobre pseudociencias}

Los diferentes ítems del cuestionario diseñado señalan puntuaciones muy diversas. De manera general, la media de todas las cuestiones sin tener en cuenta el género obtuvo un valor de 2,32; es decir más próxima al desacuerdo y por tanto señalando ser escéptico ante las diferentes afirmaciones pseudocientíficas. Debemos destacar algunos ítems con valores muy bajos para ambos sexos con calificaciones que rondan 1 (muy en desacuerdo), tales como "Me creo todo lo que escucho” (P45) o "La mujer cuanto menos vello tenga, más tímida es.” (P23). Sin embargo, algunas afirmaciones superan la calificación de 3 (indiferente), como "La ciencia oculta la existencia de algunos animales para diversos fines" (P05) o "La ciencia no quiere aceptar cosas que sabe que en realidad son ciertas" (P42).

Las medias de los valores de ítems por temáticas según la edad de los estudiantes (en función del curso, $1^{\circ}$ o $3^{\circ}$ de ESO) no mostraron diferencias significativas (solo hubo un incremento medio de 0,04 puntos de $1^{\circ}$ a $3^{\circ}$ de ESO). En algunos ítems particulares se encontraron valores superiores en $1^{\circ}$ ESO (ítems P05, P18, P23 y P44); y en otros casos en $3^{\circ}$ ESO (ítems P01, P03, P07 y P28) con significación; pero globalmente ni por ideas pseudocientíficas, ni en superstición, ni en creencias hacia la ciencia, ni en la valoración personal del escepticismo se encuentran diferencias significativas, ni patrones claros de respuestas por curso. Tampoco se encontraron diferencias entre los dos centros educativos.

Las medias de las agrupaciones de ítems de la misma temática si arrojaron diferencias significativas en cuanto al género. La prueba t de Student determinó significación para la astrología, ufología, fisiognomía y quiromancia (Gráfico 1). Obteniendo valores superiores en chicas en astrología y quiromancia (Gráficos 2 y 3, respectivamente), con respecto a los chicos, quienes a su vez destacaron en ufología y fisiognomía (Gráficos 4 y 5, respectivamente). Sin embargo, no se encontraron diferencias significativas para el resto de los grupos: criptozoología, radiestesia, superstición, creencias hacia la ciencia y pseudociencia y, por último, el valor personal sobre el nivel personal de escepticismo. 
GRÁFICO 1. Valoración media en función del género de los grupos de items de las diversas temáticas.

Leyenda: Cript.: Criptozoología, Astr.; Astrología, Ufo.: Ufología, Radies.: Radiestesia, Fisiog.:

Fisiognomía, Quiro.: Quiromancia, Superst.: Superstición, CPC.: Creencias hacia la ciencia y pseudociencia, VPE.: Valoración personal del nivel de escepticismo” (* presenta significación estadística)

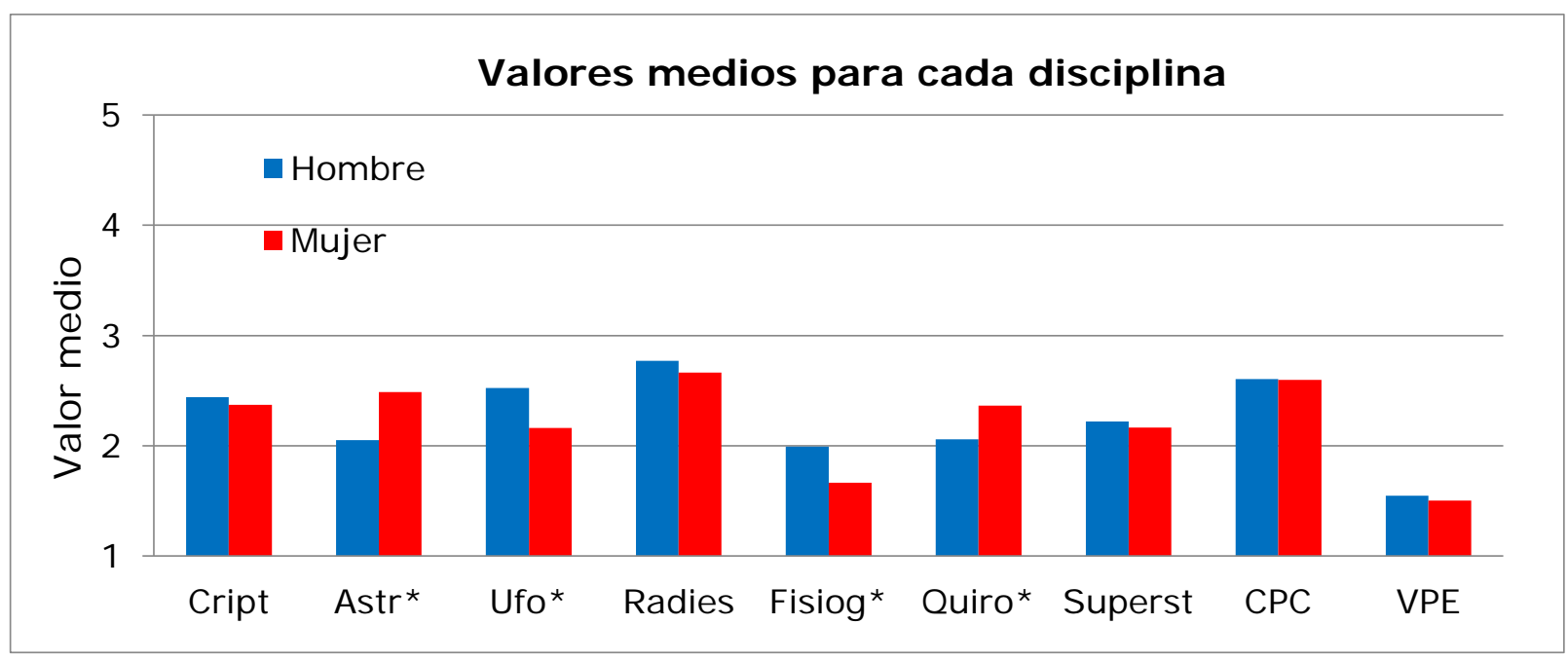

Al estudiar cada ítem de manera independiente, mediante la prueba t de Student, se encuentran diferencias significativas según el género (Gráficos 2 a 5). En las representaciones adjuntas se aprecian las medias de cada uno, señalándose la diferencia en las significativas. De esta manera, son estadísticamente significativas los ítems: "Las sirenas son reales y algunos marineros las han visto" (P03); "La ciencia oculta la existencia de algunos animales para diversos fines" (P05); "Me gusta mirar el horóscopo y creo que suele acertar" (P06); "El horóscopo suele acertar las predicciones sobre dinero, amor, trabajo o salud” (P08); "El gobierno oculta la existencia de alienígenas” (P14); “El área 51 (supuesta base militar estadounidense) realiza contactos y/o experimentación con alienígenas” (P15); “Son útiles e informativos los programas como Cuarto Milenio que suelen hablar de energías misteriosas” (P20); "El físico de una persona puede ser de ayuda para conocer su carácter" (P21); "El tamaño de la cabeza de una persona es un indicativo de su inteligencia.” (P24); "Existen personas que mirando las líneas de tus manos pueden predecir tu futuro" (P26); "Me gustaría que me leyesen la mano” (P27); "La elección de la mano (derecha o izquierda) es importante a la hora de predecir el futuro de una persona” (P29) y "Hay piedras preciosas que dan buena suerte si las llevas colgadas o cerca de ti, por ejemplo, el ágata” (P35).

No se encuentran diferencias significativas entre chicos y chicas en el resto de las cuestiones (ver enunciados en el Anexo I): P01, P02, P04, P07, P09 a P13, P16 a P19, P22, P23, P25, P28, P30 a P34, y P36 a P45.

En las gráficas observamos patrones similares en niños y niñas para todas las temáticas; aunque para algunas pseudociencias, un género es más escéptico que el otro, si bien no en todos los ítems hay significación estadística. En ufología y fisiognomía, los chicos tienen valores superiores para todos los ítems (Gráficos 4 y 5). En cambio, las chicas tienen valores superiores para todas las cuestiones de las temáticas sobre astrología y quiromancia (Gráficos 2 y 3). En criptozoología y radiestesia, superstición y creencia en la ciencia y pseudociencias, niños y niñas no presentan diferencias globales, aunque existan algunos ítems con diferencias significativas (P03, P05, P20, P35), y las valoraciones pueden ser mayores o menores según género en los diferentes ítems. 
GRÁFICO 2. Valoración media en función del género de los ítems que versan sobre astrología (*presenta significación estadística)

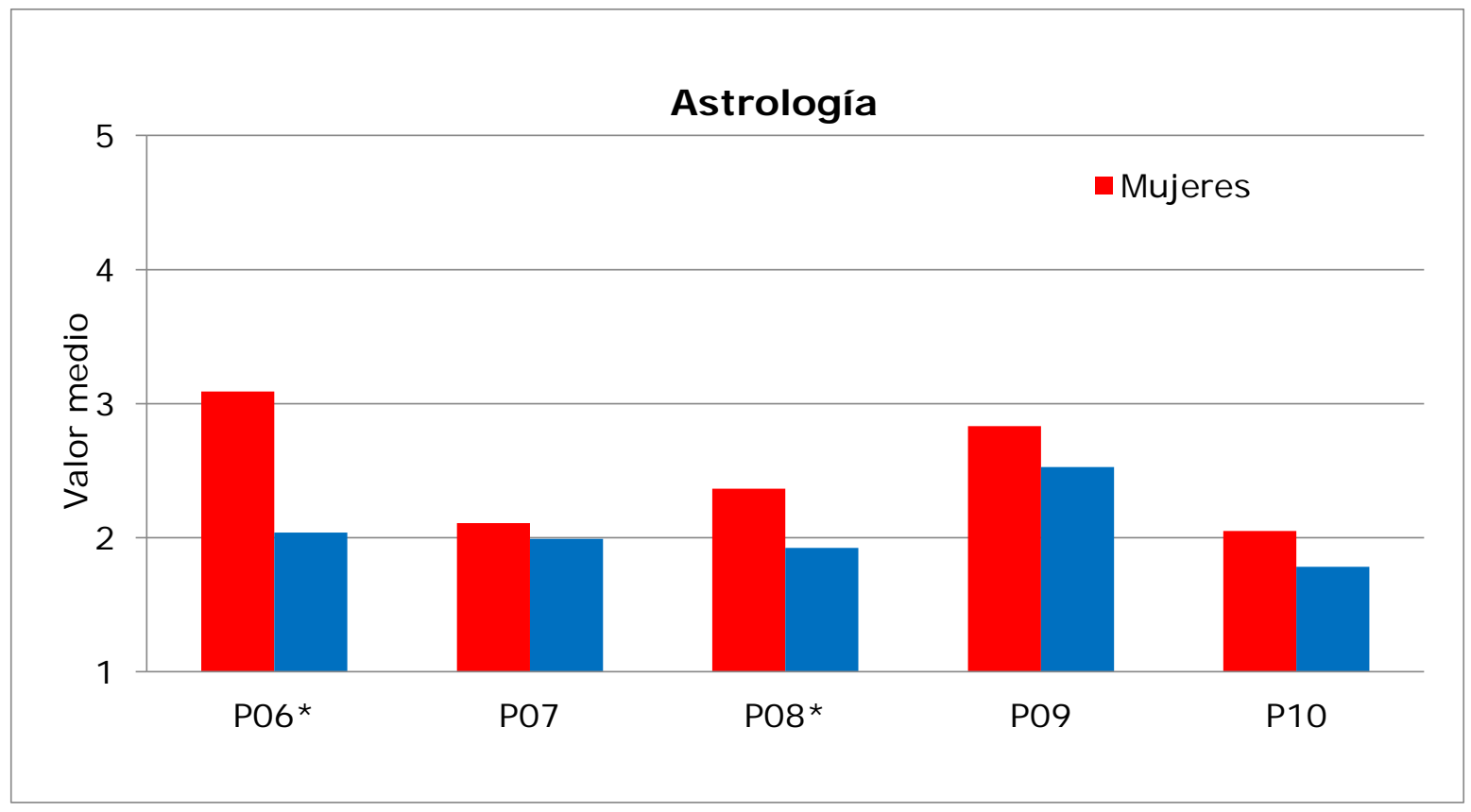

GRÁFICO 3. Valoración media en función del género de los ítems que versan sobre quiromancia (*presenta significación estadística)

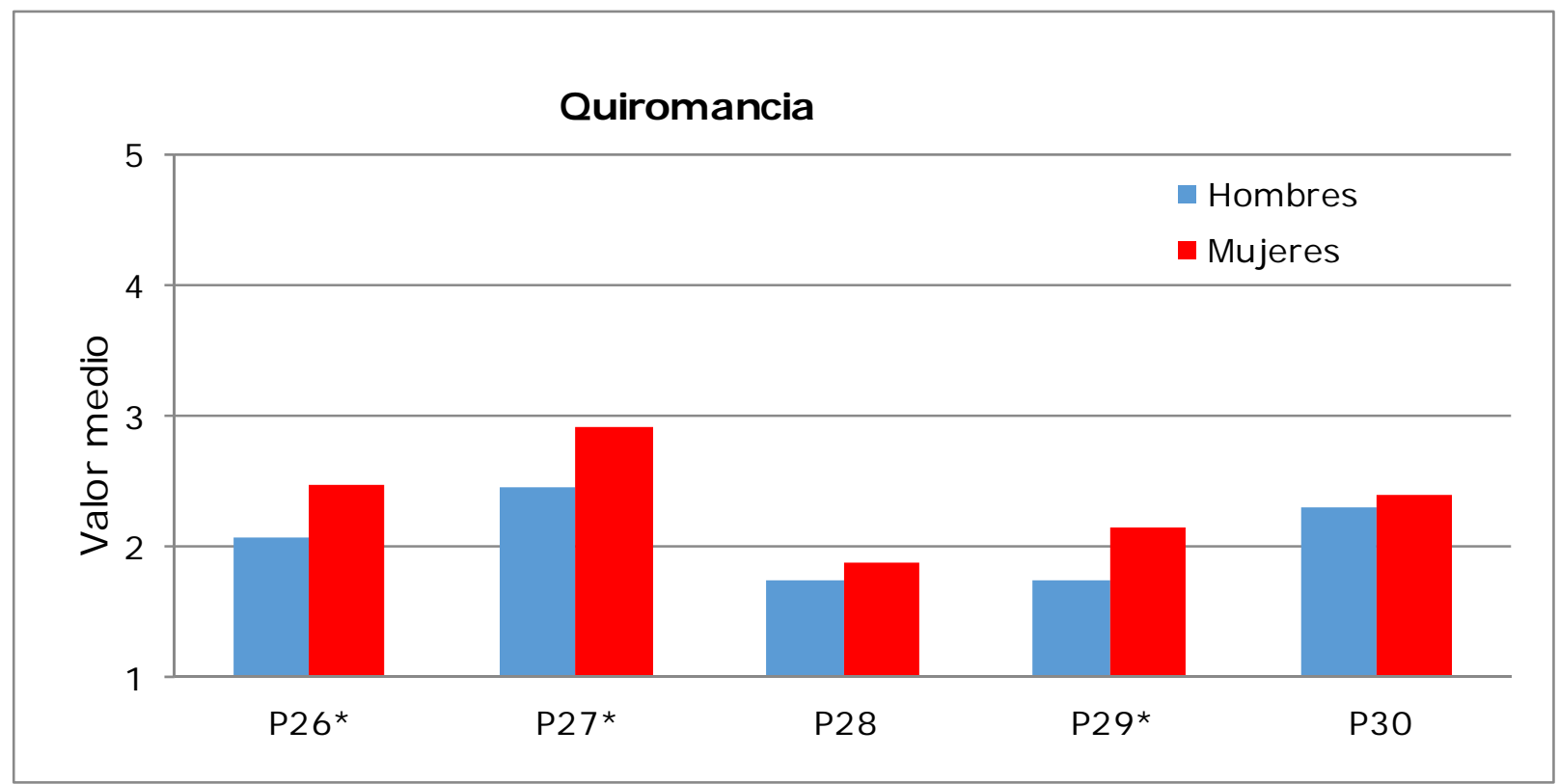


GRÁFICO 3. Valoración media en función del género de los ítems que versan sobre ufología (*presenta significación estadística)

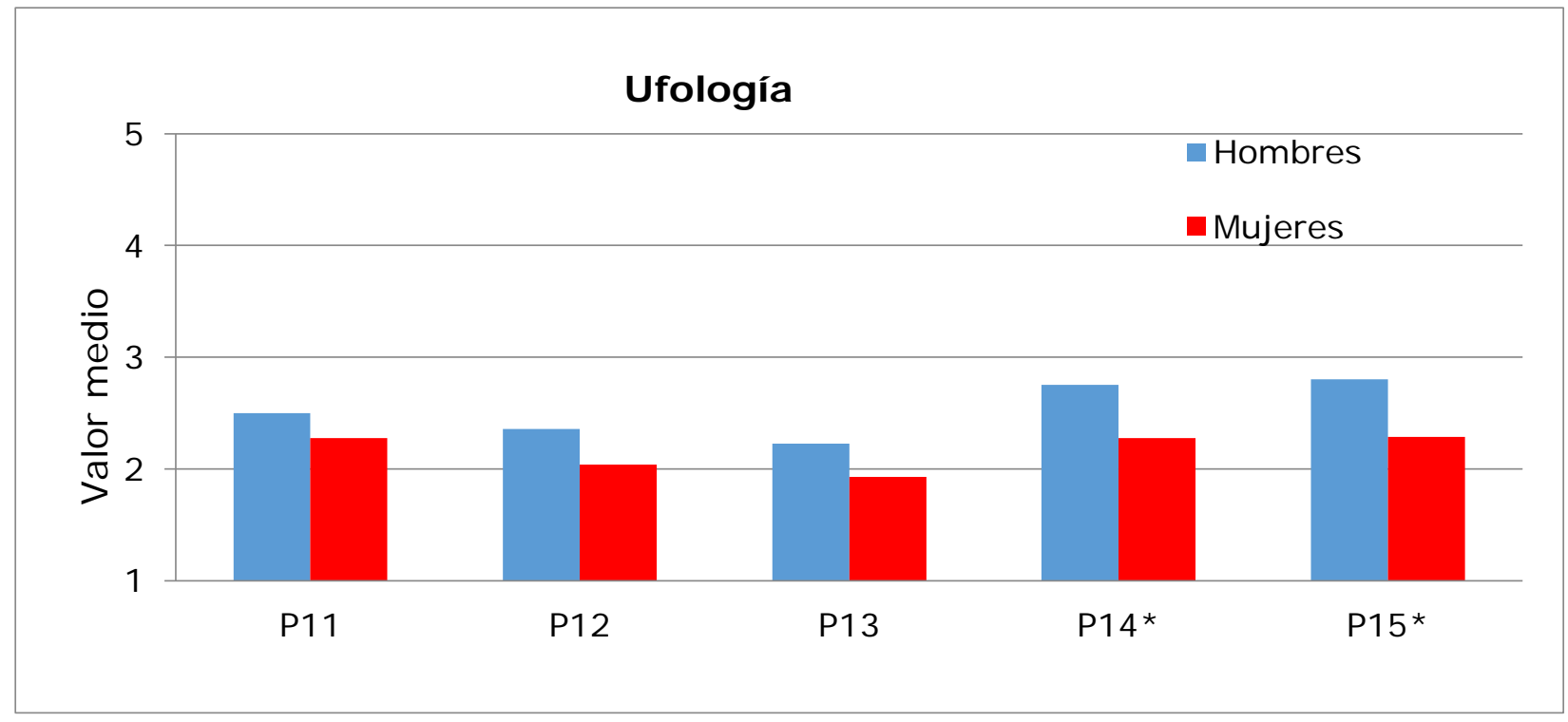

GRÁFICO 4. Valoración media en función del género de los ítems que versan sobre fisiognomía (*presenta significación estadística)

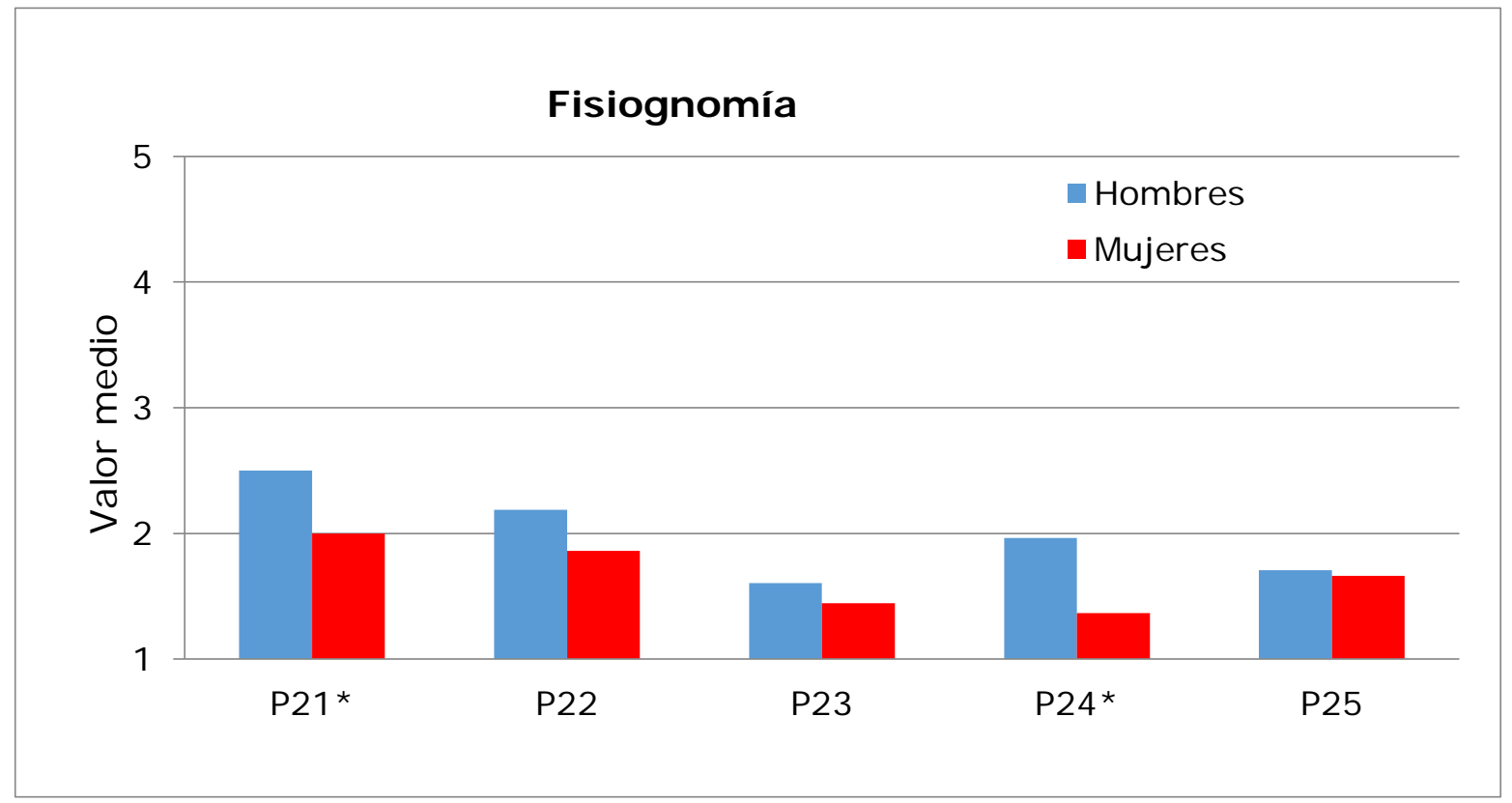

\subsection{Planteamiento didáctico en $1^{\circ}$ curso de Educación Secundaria Obligatoria}

La intervención didáctica trabajada con los alumnos de $1^{\circ}$ ESO, 12-13 años, constó de 6 actividades que se exponen a continuación, comentando y describiendo los aspectos más relevantes, así como presentado de forma ilustrativa algunos ejemplos de los trabajos realizados por los grupos de estudiantes. 
En la actividad 1 se pidió al alumnado que buscara información sobre animales y plantas descubiertos recientemente por la ciencia, fomentando el uso de diversas fuentes bibliográficas (enciclopedias, libros de texto, páginas web de instituciones científicas). Se solicitó que era necesario argumentar las posibles razones por las que se había podido retrasar el descubrimiento de cada especie.

En la Imagen 1 se muestra un ejemplo de composición realizada por los estudiantes. Todos los grupos realizaron composiciones con fotografías y elaboraron portadas y logos para ilustrar sus trabajos. La actividad resultó dinámica y se intercambió información entre los diferentes grupos de estudiantes que encontraron información de diversas especies. Muchas de las especies fueron caracterizadas como raras y curiosas por los estudiantes, generando un debate sobre la necesidad de cuidar el planeta para proteger la diversidad que alberga.

IMAGEN 1. Ejemplo de mural presentado en la realización de la Actividad 1.

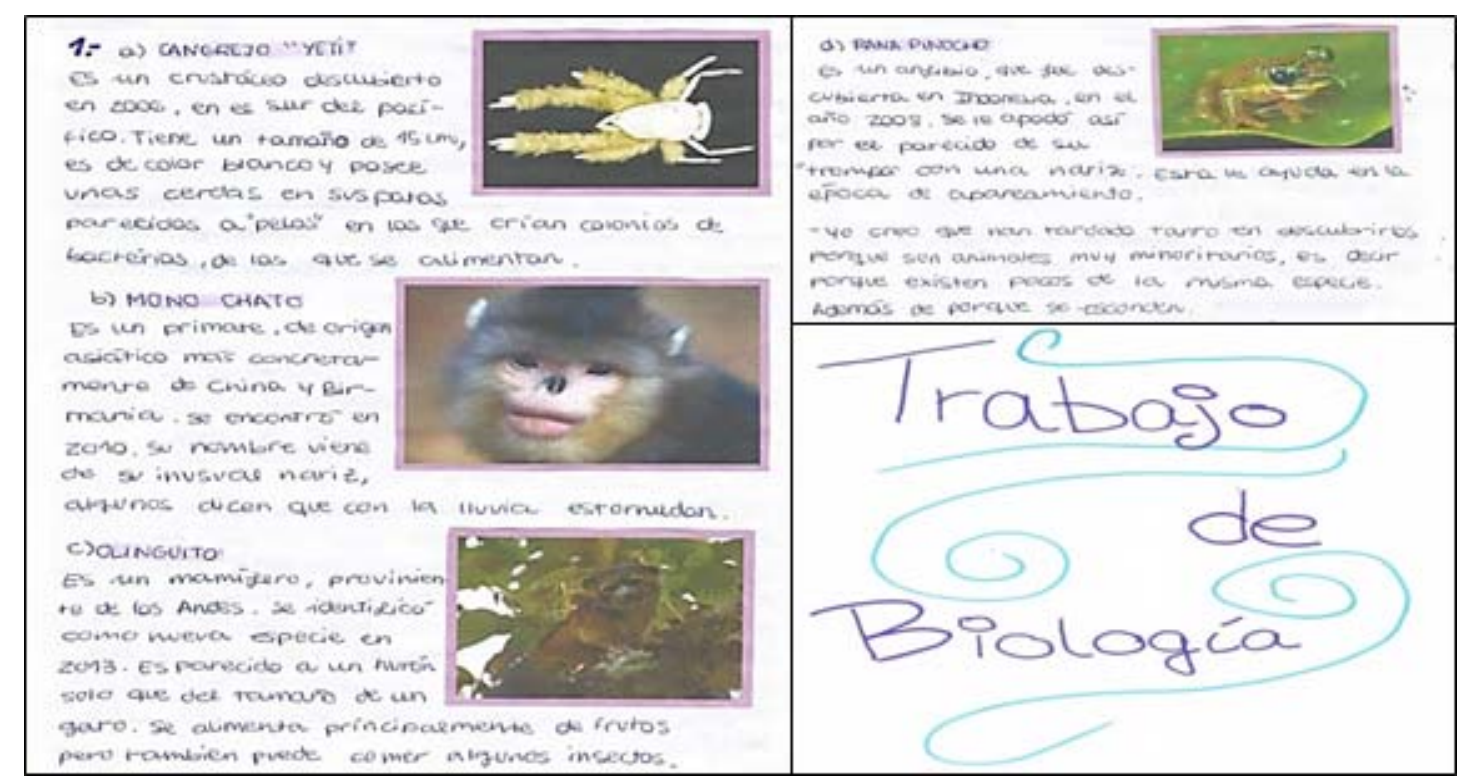

La actividad 2 planteó responder a la cuestión: ¿Por qué son falsos los horóscopos?, dando dos razones por las que debemos desconfiar de ellos. Esta actividad intentaba promover el razonamiento y la reflexión sobre la temática de la astrología, dirigiéndose especialmente a las alumnas, algunas de ellas al inicio de la actividad defendían la eficacia o verosimilitud de los pronósticos astrológicos. Recordemos que en los resultados del cuestionario la valoración de las chicas es significativamente superior a la de los chicos. Los estudiantes debatieron sus ideas y argumentaron razones para desconfiar de los horóscopos. Destacan las respuestas en las que se incide sobre la capacidad de manipulación de los horóscopos. Por ejemplo, transcribimos dos respuestas dadas por un estudiante:

- Los horóscopos solo dicen cosas nada concretas, que se aplican a cosas cotidianas de la mayoría de las personas. Por ejemplo "el día de hoy tendrás un día lleno de complicaciones” este pronóstico es poco conciso y se puede aplicar de muchas maneras en su interpretación.

- Los horóscopos pueden causar desgracias según su interpretación, ya que una persona por creer lo que dice su horóscopo puede llegar a hacer actos que pueden ocasionar cosas graves.

La actividad 3 planteaba la siguiente situación problema: "Imagínate que dos personas tienen un fuerte resfriado y deciden ir al médico. El doctor le entrega una pastilla a cada uno, sin embargo lo que no saben, es que una de las pastillas es en realidad un caramelo. Ambos salen de la consulta creyendo que su pastilla les curará su enfermedad, aunque técnicamente, solo una de ellas puede 
curar el resfriado. A los días sorprendentemente ambos están curados. ¿Qué ha podido ocurrir? Busca en diversas fuentes (Internet, enciclopedias, libros de texto, etc.) qué es el efecto placebo y explica lo que le ha sucedido al paciente que tomó el caramelo.

Los estudiantes buscaron información, principalmente en páginas WEB, para definir o comprender el efecto placebo; algunos declararon haber preguntado a sus familiares para comprender dicho efecto. La actividad trata de incidir en la comprensión del efecto placebo y su incidencia en la medicina alternativa. En la Imagen 2 se muestra la respuesta dada por un grupo de estudiantes.

IMAGEN 2. Definición de placebo y respuesta al problema planteado en la Actividad 3, según un grupo de estudiantes.

- Sustancia que carece de acción curativa pero produce un efecto terapéutico si el enfermo la toma convencido de que es un medicamento realmente eficaz; tiene el mismo aspecto, gusto y forma que un medicamento verdadero pero está hecho de productos inertes y sin ningún principio activo.

"es un hipocondríaco y el médico le receta placebos en muchas ocasiones"

- El paciente al irse convencido de que la medicina le curaría, su mente le hizo creer estar curado, pero si el paciente hubiera sabido que era un caramelo y se lo hubiera tomado no creería estar curado.

La actividad 4 planteaba una nueva situación problemática ligada a la salud y el uso de terapias alternativas, denominadas en ocasiones como medicina alternativa. Se planteó el caso de Steve Jobs (cofundador de Apple). Este personaje contemporáneo ha sido una de las personas más importantes de nuestra época, sin embargo, falleció siendo muy joven debido a un cáncer de páncreas. A pesar de que los médicos le detectaron la enfermedad bastante pronto y podría haberse curado, Steve no quiso seguir el tratamiento que le dieron los doctores. Pese a las advertencias de los médicos, Steve optó por diferentes técnicas de medicina alternativa, consultas a videntes y dietas extrañas que no lo curaron, y por lo tanto murió a la edad de 56 años.

Tras presentar esta información se pidió a los estudiantes que buscaran información, de modo similar a las actividades anteriores y dieran razones por las que la medicina alternativa supone un peligro para la salud. Y respondieran, además, a la cuestión: ¿Qué habría tenido que hacer Steve Jobs para curarse de su enfermedad?

Los alumnos debatieron que el término medicina alternativa puede producir confusión, indicaron que S. Jobs debería haber seguido el consejo de los médicos y resaltan que las terapias alternativas pueden tener efectos secundarios desconocidos. Sin embargo en tres grupos de estudiantes se imponía la idea de que algunas medicinas alternativas pueden funcionar pero que son "más difíciles de medir".

En la actividad 5 se solicitaba responder a la cuestión: ¿Piensas que debemos creer todo lo que dicen en la televisión? ¿Por qué?

En su totalidad los estudiantes eran conscientes del peligro de manipulación que suponen los medios, aunque también la mayoría admitieron creer como ciertas muchas de las informaciones que ven en la televisión o en otras fuentes como Internet. Una respuesta común que transcribimos fue: 
No, porque hay medios de comunicación social que manipulan las noticias para tener más audiencia.

Hoy en día con las nuevas tecnologías es muy fácil modificar videos, hacer montajes falsos etc para hacer creer que la noticia es verdadera aunque no lo sea.

En la actividad 6 se trabajó con un problema matemático de probabilidades: Tradicionalmente se cree que el trébol de cuatro hojas es un símbolo de buena suerte. Sin embargo, parece que en realidad se debe a una mutación que puede hacer aumentar su número de hojas (el récord son 56 hojas). El famoso trébol de cuatro hojas no da suerte, la suerte es encontrarlo ya que uno de cada 10.000 tiene cuatro hojas. Si he encontrado 3 tréboles de cuatro hojas: ¿Cuántos tréboles he mirado?

Se fomentó el trabajo con la idea de la superstición y los objetos supersticiosos; aunque hubo errores en la respuesta matemática, resaltó el interés por el origen de este tipo de trébol y poder encontrarlo en algún momento.

En conjunto las actividades permitieron el fomento del trabajo colaborativo, la consulta de diversas fuentes de información y se creó un clima de discusión y debate poco habitual en las clases de ciencias más tradicionales. No se cuantificó si hubo cambios en las valoraciones y opiniones sobre algunas de las ideas pseudocientíficas que se trataban de cuestionar con las actividades presentadas. Sin embargo, y en una valoración cualitativa, si se pudo apreciar que en temas como la astrología o las medicinas alternativas, los estudiantes se mostraron muy críticos y conscientes de las limitaciones de esas ideas. Algunas de las respuestas eran simples y sencillas mientras que otras denotaban un razonamiento personal crítico sobre las diferentes cuestiones planteadas.

\section{DisCUSIón}

Los resultados obtenidos nos presentan el nivel de escepticismo de nuestra muestra. Basados en la media de las 44 cuestiones sin distinción de género, se obtuvo un valor de 2,32 (en desacuerdo) para toda la muestra. Podríamos afirmar que el grado de escepticismo de los estudiantes es relativamente elevado, aunque menor que el que ellos mismos declaran a partir del último ítem "Me creo todo lo que escucho" (P45), pues a ellos mismos se dan una calificación más baja (valor medio de 1,5) que en el resto de ítems.

Algunos ítems obtuvieron calificaciones relativamente altas (3, indiferente, o ligeramente superior próximo al cuerdo), mostrando un cierto nivel de aceptación o credibilidad entre el alumnado. No se encontraron diferencias significativas ni por edad ni por centro educativo; en cambio se registraron diferencias a nivel de género para cada temática.

Lo habitual en los estudios sobre esta temática es la búsqueda de diferencias por edad y/o género. En el caso de la edad en nuestra muestra no se han encontrado diferencias, aunque están descritos en otros casos con estudiantes de mayor edad y en adultos (McLeish, 1984; Preece y Baxter, 2000; Shermer, 2003; Wiseman y Watt, 2004). En diversos trabajos no se encuentra una relación fuerte sino siempre muy débil entre el nivel de estudios en ciencias (en función de la edad y los cursos realizados) y el rechazo o incredulidad hacia la pseudociencia. La hipótesis planteada en la mayor parte de los trabajos es que la relación debe ser negativa (mas conocimiento de ciencia debe acompañarse a una menor creencia en pseudociencias), sin embargo tal relación no se encuentra en muchas ocasiones (Lundstrom y Jakobsson, 2009; Ryan, et al., 2004; Walker, et al., 2002) o bien la relación siempre es muy débil (Johnson y Pigliucci, 2004; McLeish, 1984; Preece y Baxter, 2000).

Es importante destacar la escasa cantidad de estudios realizados utilizando algunas de las pseudociencias aquí tratadas, siendo más habituales los estudios con astrología y ufología. En el caso de la criptozoología, no se encontró ninguna diferencia importante entre chicos y chicas a nivel de temática. Aunque las chicas demostraron mayor interés sobre la existencia de sirenas, esto se 
puede deber a que una gran parte de la publicidad o juguetes de fantasía se destinan a dicho público. En el caso de los chicos, destacaron en su desconfianza hacia el gobierno por la posibilidad de ocultar la existencia de animales. En la astrología se detectó diferencias significativas por género. Al igual que en otros estudios (Preece y Baxter, 2000), las chicas obtuvieron valores superiores, mayor credulidad con respecto a los chicos. Estos autores indican que la presencia de horóscopos en revistas y publicaciones dirigidas a las chicas puede influir en estas creencias. Para todas las cuestiones, las chicas calificaron con mayor puntuación con respecto a los chicos, aunque tan solo dos son significativos. En la intervención didáctica realizada, apreciamos que inicialmente algunas chicas se mostraban especialmente receptivas a este tipo de ideas.

En la ufología también aparecen diferencias de género; y al igual que en Preece y Baxter (2000), los chicos son menos escépticos que las chicas, para todas las cuestiones los chicos tienen mayor puntuación, aunque solo dos ítems fueron significativos. Es posible que el interés de los chicos a la ufología se deba a que existe una enorme influencia a través de las películas y videojuegos, ya que normalmente los protagonistas de estos son varones.

En la radiestesia se presenta significación sólo en un ítem pero no a nivel de temática, aunque destaca que es la pseudociencia que presenta las más elevada valoración por parte de chicos y chicas. Esto se puede deber a que es posible que el alumnado vincule conceptos o términos como energía y medicina alternativa a la práctica de la ciencia. Es en las temáticas de salud donde las pseudociencias demuestra tener mayor aceptación por la población en general (Lundstrom y Jakobsson, 2009; Uskola, 2016). Como indicamos al comentar las actividades 4 y 5, algunos estudiantes consideraban que las medicinas alternativas pueden ser eficaces pero que son "difíciles de medir". Podemos cuestionarnos si bajo esa expresión "difícil de medir” no se encontrará una deformada imagen de la ciencia, tal como se transmite habitualmente en el aula de ciencias.

En la fisiognomía, los chicos valoraron todas las cuestiones con puntuaciones superiores con respecto a las chicas. Dos de estas cuestiones fueron significativas, demostrando que los chicos juzgan a las personas más por su aspecto que las chicas. Como han demostrado varios estudios, el desarrollo personal y físico de chicos y de chicas no suele desarrollarse conjuntamente. Es posible que, a estas edades los chicos estén más preocupados de la integración en un grupo y análisis de su entorno para buscar su espacio en la sociedad (Vázquez y Manassero, 2007). En la quiromancia a nivel de temática resultó ser significativo, teniendo valores superiores en chicas con respecto a los chicos. De igual manera, todos los ítems son superiores en chicas, siendo tres significativos. Tanto la quiromancia como la astrología presentan valores superiores en chicas y ambas guardan cierta relación. Intentan explicar temáticas como el destino, amor o fortuna, existiendo muchas páginas de internet que parecen estar destinadas preferentemente a la población femenina.

La superstición no supuso diferenciación de género alguna a nivel de temática. Solo a nivel de un ítem, la suerte que otorgan ciertas piedras, en el que las chicas demostraron un mayor interés. Si podemos destacar que ambos sexos demostraron valores altos en cuanto al trébol de cuatro hojas ya que es un objeto muy conocido y codiciado. Las diferentes cuestiones posteriores sobre las creencias hacia la ciencia y las pseudociencia demuestran que los alumnos conocen los conceptos planteados. Además, no se detectó diferencias en cuanto al género.

$\mathrm{Al}$ igual que en otros estudios, globalmente no se puede afirmar que haya diferencia de género entre las creencias de niños y niñas acerca de la pseudociencia en general, sino que según qué tipo de temática consideremos se pueden encontrar diferencia por género (Lundstrom y Jakobsson, 2009; Preece y Baxter, 2000; Shermer, 2003). Finalmente, la valoración personal de su nivel de escepticismo posee los valores más bajos tanto para chicos como para chicas, no detectándose diferencias significativas en cuanto al género. 


\section{CONCLUSIONES. IMPLICACIONES EDUCATIVAS}

Los resultados globales nos indican que los estudiantes encuestados son relativamente escépticos, existiendo ciertas diferencias significativas por género a nivel de algunas pseudociencias particulares. En la astrología y la quiromancia las chicas se mostraron más crédulas; y los chicos tienen más tendencia a aceptar creencias de la ufología y la fisiognomía. En la criptozoología y radiestesia no se determinó diferencias significativas a nivel de género. Sin embargo es llamativo que el mayor nivel de aceptación de creencias pseudocientíficas se presenta en la radiestesia. Otros grupos de cuestiones sobre superstición, creencias hacia la ciencia y pseudociencia o su propio valor de escepticismo tampoco mostraron diferencias de género significativas. Por ello se puede afirmar que existe cierta relación entre el género y el pensamiento pseudocientífico y científico, ligado a temas concretos pero no de forma general. Como indican algunos autores, la influencia del género en las desigualdades son comunes en todo el mundo y en muy diversos aspectos (Maruani, 2002).

El mantenimiento de estas opiniones y su aparición ya en estas edades, hacia los 12 años, están influidas por fuertes creencias culturales y sociales en todas las sociedades humanas (Sagan, 1995). Nuestros estudiantes no mostraron diferencia significativa al avanzar por edad, la diferencia de 0,04 puntos no es significativa, y por tanto al tener cursadas más materias científicas, en todo caso eran alumnos de educación obligatoria y por tanto habían cursado las materias básicas de ciencias. Esta falta de relación o en todo caso muy débil, descrita ampliamente en la bibliografía ya citada, es un tema de preocupación en los diferentes países donde se han realizado estos trabajos (Suecia, Inglaterra, Estados Unidos, Canadá).

La contradicción que supone la aparente falta de relación entre la formación científica y la aceptación de ideas pseudocientíficas podría estar ligada a la forma tradicional de la enseñanza de las ciencias. Es habitual que no se realicen cuestiones acerca de los temas que abordan las pseudociencias y ni siquiera se plantea cuestionar su forma de trabajar; todo ello debido a que se olvida que una parte del conocimiento científico son los procesos y los contextos en que trabaja la ciencia, grandes olvidados en la alfabetización científica (Miller, 1987; Abd-El-Khalick y Lederman, 2000; Shermer, 2003).

Andrew Ede (2000) postula tres razones por las cuales la enseñanza de la ciencia no favorece el pensamiento crítico y racional sino que incluso puede disuadirlo o disminuirlo. Así, critica la búsqueda de una única respuesta correcta en muchas actividades de laboratorio, la falta de contextualización de las actividades y el exceso de aura de expertos al presentar resultados. En definitiva, los estudiantes terminan su preparación científica en una posición de aceptar o rechazar afirmaciones basándose en lo que se les dice que crean, en lugar de ser capaces de poder evaluar críticamente las evidencias que se les presentan. Es posible que un estudiante acumule muchos conocimientos de ciencias, pero no aprenda a distinguir correctamente qué es ciencia y qué no. Otros autores sugieren que a los estudiantes se les enseña qué pensar pero no cómo pensar (Walker et al., 2002)

Nosotros hemos planteado una serie de cuestiones a alumnos de $1^{\circ}$ ESO y su respuesta fue muy positiva, valorándose en especial que se alejaban de la respuesta única que se suele dar en el aula de ciencias; así como hacer crítica y poner en cuestión temáticas cotidianas que no se tratan habitualmente en el aula.

Resaltar particularmente el planteamiento de actividades en que es necesario buscar información de fuentes diversas, tanto fuentes bibliográficas tradicionales (enciclopedias y manuales clásicos que tienen el peligro de quedar olvidados en las bibliotecas de los centros escolares) como la consulta de diversas fuentes en Internet siendo cuidadoso en su selección y lectura.

Se requieren de más investigaciones contrastadas y evidentemente es necesario evaluar de forma más amplia estas actividades, así como que las mismas se generalicen dentro del currículo de 
ciencias para poder encontrar una respuesta positiva a la aparente contradicción que se aprecia en la poca relación entre conocimiento científico y rechazo a la pseudociencia. Dar una oportunidad al desarrollo del pensamiento crítico, incluso desde los niveles básicos de la enseñanza, puede ser una respuesta a este problema. Necesitamos generalizar este tipo de trabajo crítico y abierto en el aula de ciencias para evitar que los estudiantes confundan ideas, siendo importante que las aulas sirvan de contraste a otras fuentes de información particularmente proclives a la confusión interesada o no.

\section{Referencias}

Abd-El-Khalick, F. y Lederman, N.G. (2000). Improving science teachers' conceptions of nature of science: A critical review of the literatura. International Journal of Science Education, 2287, 665-701. DOI: 10.1080/09500690050044044

Alemañ Berenguer, R.A. (2013). Ciencia e irracionalidad en la cultura de masas. En Manual Formativo de ACTA (Autores Científico-Técnicos y Académicos). Edición Digital.

Azpiazu, L. (2004). Un zahorí hecho a sí mismo. DYNA, 79(4), 47-50.

Caro Baroja, J. (1988). Historia de la fisiognómica: el rostro y el carácter. Madrid: Ediciones AKAL.

CBS. (2002). Most believe in psychic phenomena. Recuperado de: http://www.cbsnews.com/stories/2002/04/29/opinion/polls/main507515.shtml

CE. (2005). Europeans, science and technology Eurobarometer 2005 Recuperado de: http://ec.europa.eu/public_opinion/archives/ebs/ebs_224_report_en.pdf

Eckblad, M. y Chapman, L. J. (1983). Magical ideation as an indicator of schizotypy. Journal of consulting and clinical psychology, 51(2), 215-225. DOI: 10.1037/0022-006X.51.2.215

Ede, A. (2000). Has Science Education Become an Enemy of Scientific Rationality? Skeptical Inquirer, 24, 48-51.

Gámez, L. A. (2002). Los periodistas y las falsas ciencias. Mediatika. Cuadernos de Medios de Comunicación, 8, 27-37.

Goode, E. (2002). Education, scientific knowledge, and belief in the paranormal. Skeptical Inquirer, 26(1), 24-27.

Guarín, R. B. (2017). Medicinas alternativas: perpetuación de la ignorancia o utilización de información histórica. Revista Salud UIS, 49(3), 416-418. Recuperado de: http://www.redalyc.org/articulo.oa?id=343855203001

Heuvelmans, B. (1969). Note preliminaire sur un specimen conserve dans la glace, d'une forme encore inconnue d'hominide vivant Homo pongoides (sp. seu subsp. nov.). Bulletin de I'Institut Royal des Science Naturelles de Belgique, 45, 1-24.

Johnson, M., y Pigliucci, M. (2004). Is knowledge of science associated with higher skepticism of pseudoscientific claims? American Biology Teacher, 66(8), 536-548. Recuperado de: https://trace.tennessee.edu/utk_chanhonoproj/659

Lundstrom, M. y Jakobsson, A. (2009). Students' Ideas Regarding Science and Pseudo-science in Relation to the Human Body and Health. Nordina, 5 (1), 3-17.

Marbà Tallada, A. y Márquez Bargallo, C. (2010). ¿Qué opinan los estudiantes de las clases de ciencias? Un estudio transversal de sexto de primaria a cuarto de ESO. Enseñanza de las Ciencias, 28(1), 19-30. Recuperado de: https://www.raco.cat/index.php/Ensenanza/article/view/189093

Maruani, M. (2002). Trabajo y el empleo de las mujeres. Madrid: Editorial Fundamentos.

McLeish, J. (1984). Children’s superstitions: British and Canadian. Canadian Journal of Education, 19(4), 425-436.

Miller, J. D. (1987). The scientifically illiterate. American Demographics, 9, 27-31. 
Morrone, J. J. y Fortino, A. D. (1996). La zoología de los animales fantásticos: Apuntes para un bestiario criptozoológico. Revista Museo (La Plata) 2(8), 75-80.

NSF. (2006). Science and engineering indicators 2004: National Science Foundation. Recuperado de: http://www.nsf.gov/statistics/seind04

Preece, P. F. y Baxter, J. H. (2000). Scepticism and gullibility: The superstitious and pseudoscientific beliefs of secondary school students. International Journal of Science Education, 22(11), 1147-1156. DOI: 10.1080/09500690050166724

Rodríguez Hidalgo, I. (2005). Amores horoscopales. El Escéptico, 20, 50-51.

Ryan, T. J., Brown, J., Johnson, A., Sanberg, C., y Schildmier, M. (2004). Science literacy and belief in the paranormal-an empirical test. Skeptic, 10(4), 12-13.

Sabadell, M. Á. (1993). ¿Está escrito en las estrellas? Una revisión crítica de la astrología. $L a$ Alternativa Racional, 30, 5-22.

Sagan, C. (1995). The demon-haunted world: Science as a candle in the dark. New York: Random House.

Shermer, M. (2003). Why smart people believe weird things. Skeptic, 10(2), 62-73.

Suberbiola, X. P. y Bardet, N. (1998). El arca de Noé de los seres extraordinarios: una aproximación a las manifestaciones excéntricas de las ciencias naturales. Criptozoología, futurozoología y parabiología. En Evolucionismo y racionalismo (pp. 315-326). Institución Fernando el Católico.

Tobacyk, J. J. (2004). A revised paranormal belief scale. International Journal of Transpersonal Studies, 23(1), 94-98. DOI: 10.24972/ijts.2004.23.1.94

Uskola, A. (2016). ¿Los productos homeopáticos pueden ser considerados medicamentos?: Creencias de maestras/os en formación. Revista Eureka sobre Enseñanza y Divulgación de las Ciencias, 13 (3), 574-587. DOI: /10.25267/Rev_Eureka_ensen_divulg_cienc.2016.v13.i3.05

Uskola, A. (2017). Escepticismo del profesorado de primaria en formación hacia las pseudociencias: influencia de las concepciones erróneas en el caso de la homeopatía. Profesorado, Revista de Curriculum y formación del profesorado, 21 (2), 391-408.

Vázquez, A. y Manassero, M. A (2007). Las actividades extraescolares relacionadas con la ciencia y la tecnología. Revista Electrónica de Investigación Educativa, 9(1), 1-34. Recuperado de: http://redie.uabc.mx/vol9no1/contenido-vazquez3.html

Vázquez, A. y Manassero, M. A (2009). La relevancia de la educación científica: actitudes y valores de los estudiantes relacionados con la ciencia y la tecnología. Enseñanza de las Ciencias, 27(1), 33-48. Recuperado de: https://www.raco.cat/index.php/Ensenanza/article/view/132205

Walker, W. R., Hoekstra, S. J., y Vogl, R. J. (2002). Science education is no guarantee of skepticism. Skeptic, 9(3), 24-28.

Webster, R. (2000). Palm Reading for Beginners: Find Your Future in the Palm of Your Hand. Minnesota: Llewellyn Worldwide.

Wiseman, R., y Watt, C. (2004). Measuring superstitious belief: Why lucky charms matter. Personality and Individual Differences, 37, 1533-1541.

\section{CóMO CITAR ESTE ARTÍ́CULO}

Quevedo-Ortiz, G., González-García, F. y Fernández-Ferrer. G. (2019). Un estudio sobre pensamiento pseudocientífico en estudiantes de educación secundaria. Didáctica de las ciencias experimentales y sociales, 37, 147-164. DOI: 10.7203/DCES.37.15339 


\section{Anexo I. Cuestionario sobre pseudociencias}

Marca lo que corresponda: Mujer.

Hombre

Curso IES

Señala en una escala del 1 al 5 el grado de acuerdo en relación a las siguientes afirmaciones. Teniendo en cuenta que 1 es muy en desacuerdo, 2 en desacuerdo, 3 indiferente, 4 de acuerdo, 5 muy de acuerdo.

\begin{tabular}{|c|c|c|c|c|c|c|}
\hline $\mathbf{N}^{\mathbf{0}}$ & Preguntas & 1 & 2 & 3 & 4 & 5 \\
\hline 1 & Buscaría al monstruo del lago Ness & & & & & \\
\hline 2 & Los mamuts aún existen aunque digan que están extintos & & & & & \\
\hline 3 & Las sirenas son reales y algunos marineros las han visto & & & & & \\
\hline 4 & $\begin{array}{l}\text { El megalodón (tiburón prehistórico de más de } 20 \text { metros y } \\
\text { clasificado como extinto) habita en las profundidades oceánicas }\end{array}$ & & & & & \\
\hline 5 & $\begin{array}{l}\text { La ciencia oculta la existencia de algunos animales para diversos } \\
\text { fines }\end{array}$ & & & & & \\
\hline 6 & Me gusta mirar el horóscopo y creo que suele acertar & & & & & \\
\hline 7 & $\begin{array}{l}\text { Nuestras acciones, comportamientos y actitudes dependen de } \\
\text { alguna manera de los astros del universo }\end{array}$ & & & & & \\
\hline 8 & $\begin{array}{l}\text { El horóscopo suele acertar las predicciones sobre dinero, amor, } \\
\text { trabajo o salud }\end{array}$ & & & & & \\
\hline 9 & $\begin{array}{l}\text { Las características de una persona suelen coincidir con las de su } \\
\text { signo del zodiaco (Aries es entusiasta y ambicioso, etc...) }\end{array}$ & & & & & \\
\hline 10 & El signo del zodiaco de tu pareja puede influir en la relación & & & & & \\
\hline 11 & $\begin{array}{l}\text { Los alienígenas existen y a veces han visitado la Tierra porque hay } \\
\text { gente que los ha visto }\end{array}$ & & & & & \\
\hline 12 & $\begin{array}{l}\text { Cada vez son más comunes los avistamientos de platillos volantes } \\
\text { en los cielos de todo el mundo }\end{array}$ & & & & & \\
\hline 13 & $\begin{array}{l}\text { Los extraterrestres han ayudado al desarrollo de antiguas } \\
\text { civilizaciones humanas }\end{array}$ & & & & & \\
\hline 14 & El gobierno oculta la existencia de alienígenas & & & & & \\
\hline 15 & $\begin{array}{l}\text { El área } 51 \text { (supuesta base militar estadounidense) realiza contactos } \\
\text { y/o experimentación con alienígenas }\end{array}$ & & & & & \\
\hline
\end{tabular}




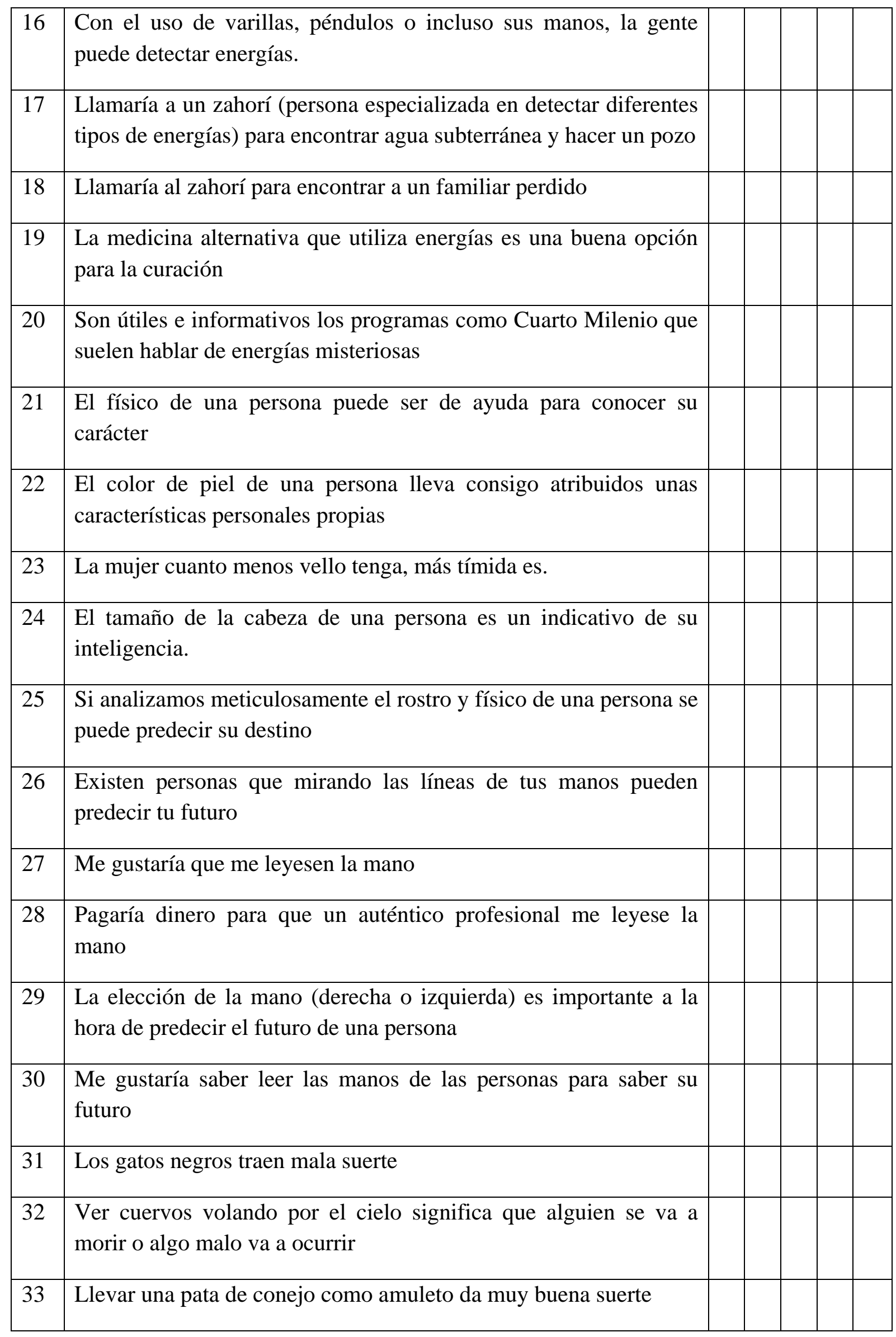




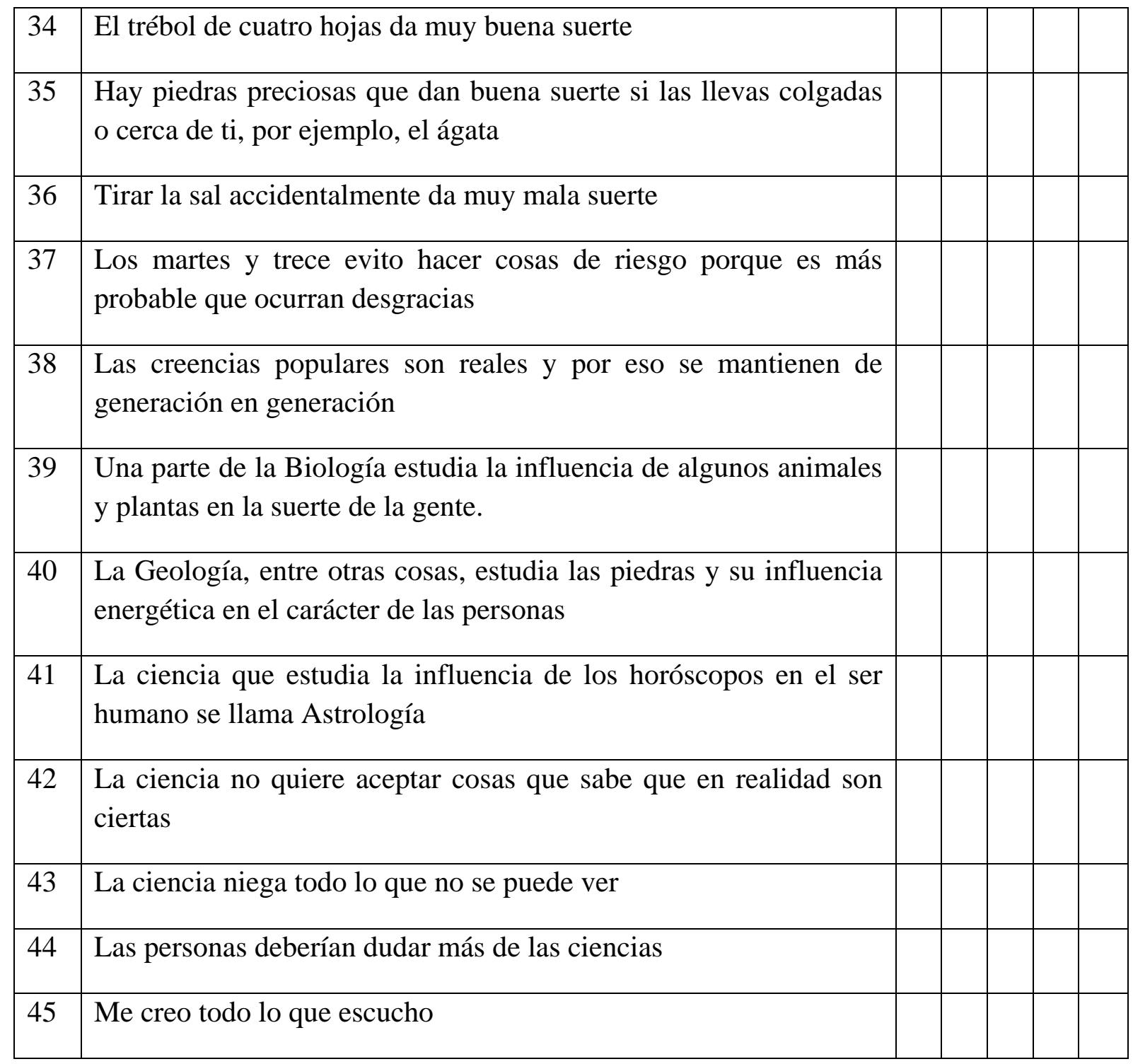

